\title{
Non-smooth homoclinic bifurcation in a conceptual climate model
}

\author{
JULIE LEIFELD \\ Department of Mathematics, University of Minnesota, Minneapolis, MN, USA \\ email:leif0020@umn.edu
}

(Received 24 June 2017; revised 4 March 2018; accepted 6 March 2018; first published online 5 April 2018)

\begin{abstract}
Collision of equilibria with a splitting manifold has been locally studied, but might also be a contributing factor to global bifurcations. In particular, a boundary collision can be coincident with collision of a virtual equilibrium with a periodic orbit, giving an analogue to a homoclinic bifurcation. This type of bifurcation is demonstrated in a non-smooth climate application. Here, we describe the non-smooth bifurcation structure, as well as the smooth bifurcation structure for which the non-smooth homoclinic bifurcation is a limiting case.
\end{abstract}

Key words: $34 \mathrm{~A} 36,34 \mathrm{~B} 60,34 \mathrm{C} 23,37 \mathrm{G} 15$

\section{Introduction}

Recently, a lot of work has gone into classifying non-smooth bifurcations. In particular, bifurcations in two-dimensional piecewise smooth systems are believed to be well understood, and have been described in $[2,4,7,8,13,15]$. Here, we look at a particular type of piecewise smooth bifurcation, called border collision. This bifurcation occurs when an equilibrium point collides with a curve of discontinuity, or splitting manifold. If the equilibrium is a node, the existing literature has focussed on whether the equilibrium persists or is annihilated by the collision. However, Filippov analysis of a conceptual climate model, called Welander's model, shows that there are other interesting questions to be asked about border collision bifurcations. In some cases, the node may persist but change stability, implying a fundamental change in the behaviour of the system. In this case, it is necessary to explore the global dynamics of the system to gain a full understanding. In Welander's model, this change in stability occurs simultaneously to a homoclinic explosion, in which infinitely many homoclinic orbits originate from the boundary equilibrium. These homoclinic orbits are also not of similar ilk to many other homoclinic orbits seen in non-smooth bifurcation classification, as they do not involve grazing of periodic orbits with splitting manifolds $[4,8,13,15]$. As the system moves through the bifurcation, the homoclinic orbits are instantaneously destroyed, leaving behind a large periodic orbit. After briefly describing some necessary vocabulary in Section 2, the climate model will be introduced in Section 3, and both a local and global analysis of the model will be discussed in Sections 4 and 5. Finally, a description of the smooth phenomena, which limits to the non-smooth bifurcation, will be undertaken in Section 6 . 


\section{Filippov analysis of non-smooth systems}

It is necessary to include a brief note about notation. A piecewise smooth system can be written in the form as

$$
\dot{x}=f(x, \lambda)
$$

with $f$ being a vector function depending on the variable $x$ and a non-smooth function $\lambda$, where $\lambda$ is expressed as

$$
\lambda=\left\{\begin{array}{ll}
1 & h(x)>0 \\
0 & h(x)<0
\end{array},\right.
$$

where $h(x)$ is a scalar function, which defines the location of the discontinuity curve, or splitting manifold.

Behaviour of the system away from the splitting manifold can be determined using classical methods, but along the splitting manifold new dynamics must be defined. Here, we use Filippov's convex combination method [7]. If the vector fields on either side of the splitting manifold point in the same direction, one expects trajectories there to cross, and we call this a crossing region. Alternatively, if both the vector fields point either toward or away from the splitting manifold, trajectories slide along the manifold, and we call this a sliding region. Sliding regions exist when the system of equations

$$
\begin{aligned}
S=f(x ; \lambda) \cdot \nabla h(x) & =0 \\
h(x) & =0
\end{aligned}
$$

can be solved for $\lambda \in[0,1]$. When $f(x, \lambda)$ has a linear dependence on $\lambda$, this corresponds to locations for which the convex combination of the vector fields contains a vector in the direction of the splitting manifold. Stability of the splitting manifold itself can be found using 2.3. If

$$
\frac{d}{d \lambda} S<0
$$

the sliding region is stable, and if

$$
\frac{d}{d \lambda} S>0
$$

the sliding region is unstable.

Sliding flow along the splitting manifold can be determined by solving (2.3) for $\lambda^{*}(x)$, then looking at the dynamics, we have

$$
\dot{x}=f\left(x, \lambda^{*}(x)\right) \text {. }
$$

Dynamical phenomena from (2.4) can then be determined in the normal way. Equilibria of equation (2.4) are called pseudo-equilibria. These pseudo-equilibria are pseudo-nodes if they are nodes in the Filippov flow and their Filippov stability agrees with the stability of the sliding region. Otherwise, they are called pseudo-saddles. 


\section{The conceptual climate model}

Welander's model [19] is an ocean convection model, given here in non-dimensionalized form

$$
\begin{gathered}
\dot{T}=1-T-k(\rho) T, \\
\dot{S}=\beta(1-S)-k(\rho) S, \\
\rho=-\alpha T+S,
\end{gathered}
$$

where $T$ and $S$ are temperature and salinity of the surface ocean, and $\rho$ is water density, given by a linear combination of the variables. $k(\rho)$ is a convective mixing function, which is assumed to be small when $\rho$ is small, and large when $\rho$ is large. Welander gives two specific functions for $k(\rho)$

$$
k(\rho)=\frac{1}{\pi} \tan ^{-1}\left(\frac{\rho-\varepsilon}{a}\right)+\frac{1}{2},
$$

and the pointwise limit,

$$
k(\rho)=\left\{\begin{array}{ll}
1 & \rho>\varepsilon \\
0 & \rho<\varepsilon
\end{array} .\right.
$$

Here, we focus on the non-smooth model, although the corresponding smooth model will be discussed in Section 6. The model given here is the non-dimensionalized version of Welander's model. The variables here should be physically interpreted as deviations from a constant temperature and salinity forcing delivered by the atmosphere. The parameter range of the bifurcation here is not wildly different from the parameter range used by Welander, but the reader should refer to [19] for more specifics about the physical interpretation of the model. Welander's model was relevant at the time of its publication for displaying a large, internally forced oscillation, without any oscillatory forcing. The bifurcation discussed here is related to the oscillation seen by Welander.

Welander's model has three parameters: $\alpha, \beta$ and $\varepsilon$. We will use Welander's values for $\alpha$ and $\beta$ :

$$
\alpha=\frac{4}{5}
$$

and

$$
\beta=\frac{1}{2}
$$

$\varepsilon$ will be the bifurcation parameter. Because the location of the discontinuity in equation (3.3) is a function of $\varepsilon$, we will also do a preliminary co-ordinate change

$$
\begin{aligned}
& x=T, \\
& y=S-\alpha T-\varepsilon,
\end{aligned}
$$


giving a new system

$$
\begin{aligned}
\dot{x} & =1-x-k(y) x, \\
\dot{y} & =\beta-\beta \varepsilon-k(y) \varepsilon-\alpha-(\beta+k(y)) y-(\alpha \beta-\alpha) x, \\
k(y) & =\left\{\begin{array}{ll}
1 & y>0 \\
0 & y<0
\end{array} .\right.
\end{aligned}
$$

Because this co-ordinate change is linear, it causes no difficulties in the Filippov analysis of the system. In this case, $k$ is of the form of equation (2.2) in Section 2. Welander noted that for small, negative $\varepsilon$, the non-smooth system contains two stable virtual equilibria, i.e., the equilibria for $k=1$ does not exist on the side of the splitting manifold where $y>0$, and vice versa. The stability of these virtual equilibria is parameter independent. Attraction to the virtual equilibria, combined with switching across the splitting manifold, gives rise to a periodic orbit for certain values of $\varepsilon$. However, this periodic orbit does not exist for all values of $\varepsilon$. At $\varepsilon=0$, a fused focus bifurcation occurs, in which the sliding region changes stability, birthing a stable periodic orbit. One might also expect some bifurcation structure pertaining to collision of a virtual equilibrium with the splitting manifold. In particular, if one of the virtual equilibria in Welander's model crosses the splitting manifold, it should become a global attractor, influencing the global dynamics. In fact, this does occur in an $\varepsilon$ range, in a bifurcation that destroys the periodic orbit. We will look at this bifurcation locally and globally in the next sections.

\section{Border collision in the model}

Oscillation in Welander's model is predicated on attraction to two different virtual equilibria. However, it is immediately clear that parameter changes can transition these virtual equilibria into globally stable, real equilibria. The location of the virtual equilibria are given by the following system of equations:

$$
\begin{aligned}
& x=\frac{1}{1+k}, \\
& y=\frac{\beta-(\beta+k) \varepsilon-\alpha-(\alpha \beta-\alpha) x}{\beta+k} .
\end{aligned}
$$

This gives, for $k=1$,

$$
(x, y)=\left(\frac{1}{2},-\frac{1}{15}-\varepsilon\right),
$$

and for $k=0$,

$$
(x, y)=\left(1, \frac{1}{5}-\varepsilon\right) .
$$

The $x$ locations of the equilibria are fixed, but the equilibria are located on the splitting manifold for

$$
\varepsilon=\frac{1}{5}
$$


and

When

$$
\varepsilon=-\frac{1}{15}
$$

$$
0<\varepsilon<\frac{1}{5}
$$

solutions are attracted to a globally stable pseudo-equilibrium. So, although the equilibrium boundary collision at

$$
\varepsilon=\frac{1}{5}
$$

is interesting, it does not qualitatively change the dynamics of the system. Instead, the pseudo-equilibrium escapes the splitting manifold and becomes a more classically recognizable, real equilibrium. This scenario is described as a border node of the first type in [13].

However, there is a fused focus Hopf bifurcation, which occurs when $\varepsilon=0$. The stable pseudo-equilibrium becomes unstable, giving rise to a stable periodic orbit. This makes the dynamics of the second border collision 4.5 more globally interesting. For convenience, we will define

$$
\varepsilon_{0}=-\frac{1}{15}
$$

In the interest of facilitating the discussion of the global bifurcation, we will look at the boundary collision in a non-standard direction, as a pseudo-equilibrium, which becomes real when it escapes the splitting manifold through an endpoint of a sliding region. The local bifurcation is shown in Figure 1. Because the pseudo-equilibrium is unstable for $\varepsilon_{0}<\varepsilon<0$, when it escapes the sliding region at $\varepsilon_{0}$, the equilibrium also changes stability. In [13], this local scenario is not discussed, as it is claimed that, with full generality, the only two scenarios possible in a boundary node collision are annihilation and persistence, without any stability change. The following theorems address the existence and stability of the equilibrium and pseudo-equilibrium as the system moves through the bifurcation.

Theorem 4.1 For $\varepsilon_{0}<\varepsilon<0$, there exists a unique unstable pseudo-node.

Proof Stability of the sliding region is given by

$$
\left.\frac{d}{d \lambda}(f \cdot \nabla h)\right|_{y=0}=\left.\frac{d}{d k}(\beta-\beta \varepsilon-k \varepsilon-\alpha-(\beta+k) y-(\alpha \beta-\alpha) x)\right|_{y=0}=-\varepsilon .
$$

So, for $\varepsilon<0$, the sliding region is unstable. We must show that the sliding region contains a unique pseudo-equilibrium, and that pseudo-equilibrium is unstable. We first show that it is unique.

Pseudo-equilibria are found on regions of the splitting manifold where the vector fields are co-linear. This gives a condition on $x$ and $\varepsilon$

$$
-10 \varepsilon-3 x+5 x \varepsilon+4 x^{2}=0
$$

So, we need to show that for $\varepsilon_{0}<\varepsilon<0$, there is only one solution to this equation inside the sliding region. This result can be clearly seen by plotting equation (4.7) and the 


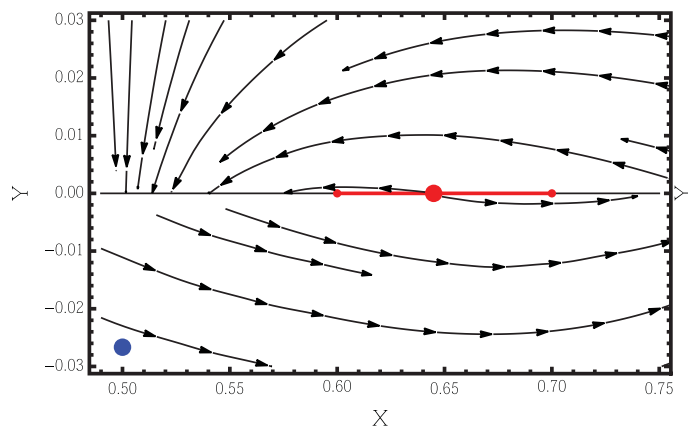

(a)

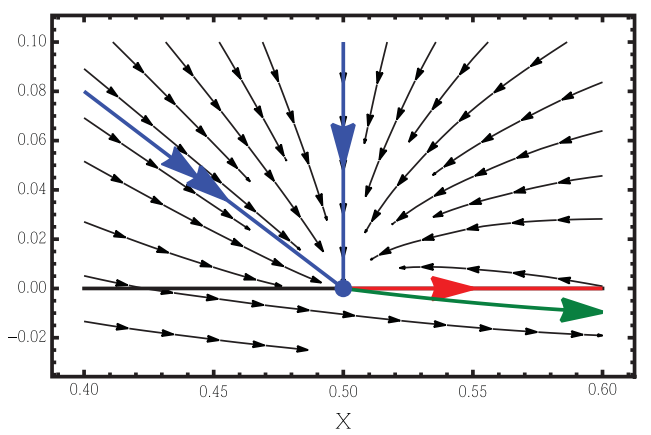

(b)

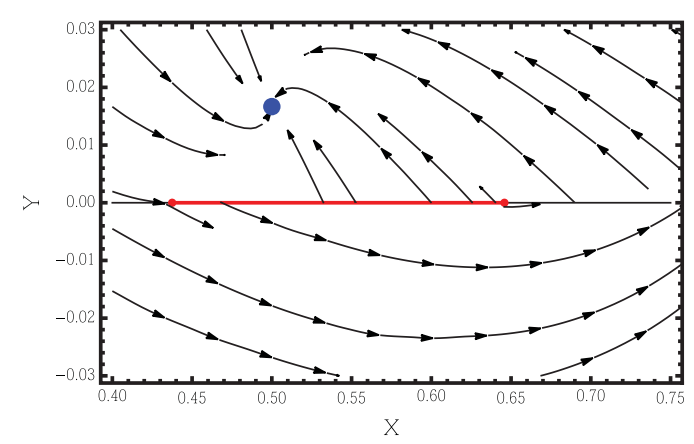

(c)

FIGURE 1. Local bifurcation structure. (a) here, $\varepsilon=-\frac{1}{25}$. In the $y>0$ region solutions are attracted to the virtual equilibrium. The sliding region, shown in red, contains an unstable pseudo-equilibrium; (b) the border collision occurs at $\varepsilon=-\frac{1}{15}$. Blue lines are in the direction of the eigenvectors. The red line is the hyperbolic trajectory away from the equilibrium along the sliding region, and the green curve is the trajectory into the $y<0$ region, which leaves the equilibrium in finite time; (c) here, $\varepsilon=-\frac{1}{12}$, the virtual equilibrium has become a stable real equilibrium. The sliding region contains no pseudo-equilibria.

bounds of the sliding region, as shown in Figure 2; however, for the sake of rigor, we prove uniqueness below.

We can rewrite equation (4.7) as follows:

$$
\varepsilon=\frac{4 x^{2}-3 x}{10-5 x}
$$

Note that $x=2$ was also not a valid solution in the original equation. The $\varepsilon$ range $\varepsilon_{0}<\varepsilon<0$ corresponds to a range of $x$ values:

$$
\frac{1}{2}<x<\frac{3}{4}
$$

Therefore, because the only minimum of equation (4.8) occurs at

$$
x=2-\frac{\sqrt{10}}{2}
$$


and the asymptote occurs at $x=2$, if the pseudo-equilibrium exists, it is unique. To show that the pseudo-equilibrium exists, we employ a change of perspective, which is possible only because the bounds of the sliding region are lines in $(x, \varepsilon)$ space. First, it is obvious that a solution to equation (4.8) exists for $\varepsilon \in\left(\varepsilon_{0}, 0\right)$. The boundaries of the sliding region are given by lines in $(x, \varepsilon)$ space

$$
x=\frac{3}{4}+\frac{15}{4} \varepsilon
$$

and

$$
x=\frac{3}{4}+\frac{5}{4} \varepsilon
$$

Writing these instead as $\varepsilon$ bounds, we see that

$$
\frac{3}{4}+\frac{15}{4} \varepsilon<x<\frac{3}{4}+\frac{5}{4} \varepsilon
$$

if and only if

Analysis shows that

$$
\frac{4}{5} x-\frac{3}{5}<\varepsilon<\frac{4}{15} x-\frac{1}{5}
$$

$$
\frac{4 x^{2}-3 x}{10-5 x}>\frac{4}{5} x-\frac{3}{5}
$$

and

as long as

$$
\frac{4 x^{2}-3 x}{10-5 x}<\frac{4}{15} x-\frac{1}{5}
$$

$$
\frac{1}{2}<x<\frac{3}{4}
$$

meaning that the $\varepsilon$ given by equation (4.8) is in the correct bounds, so $x$ is a pseudoequilibrium for the given $\varepsilon$.

We now show that the pseudo-equilibrium is an unstable equilibrium of the sliding flow. The sliding flow is found by solving the system 2.3, and then using equation (2.4). In this system, we will call $\lambda^{*}(x)=k^{*}(x)$, and the flow in the sliding region is given by

$$
\dot{x}=1-x-k^{*}(x) x .
$$

The stability of the pseudo-equilibrium is then given by the sign of the derivative of the right side of this equation, evaluated at the equilibrium. The derivative is expressed as

$$
f^{\prime}(x)=1-k^{*}-\left(\frac{d k^{*}}{d x}\right) x=\frac{-3+5 \varepsilon+8 x}{-10 \varepsilon} .
$$

We have already seen that for these $\varepsilon$ values, the pseudo-equilibrium is bounded in $x$ by

$$
\frac{1}{2}<x<\frac{3}{4}
$$

This gives us a range on $f^{\prime}$

$$
\frac{1+5 \varepsilon}{-10 \varepsilon}<f^{\prime}(\varepsilon)<\frac{3+5 \varepsilon}{-10 \varepsilon}
$$




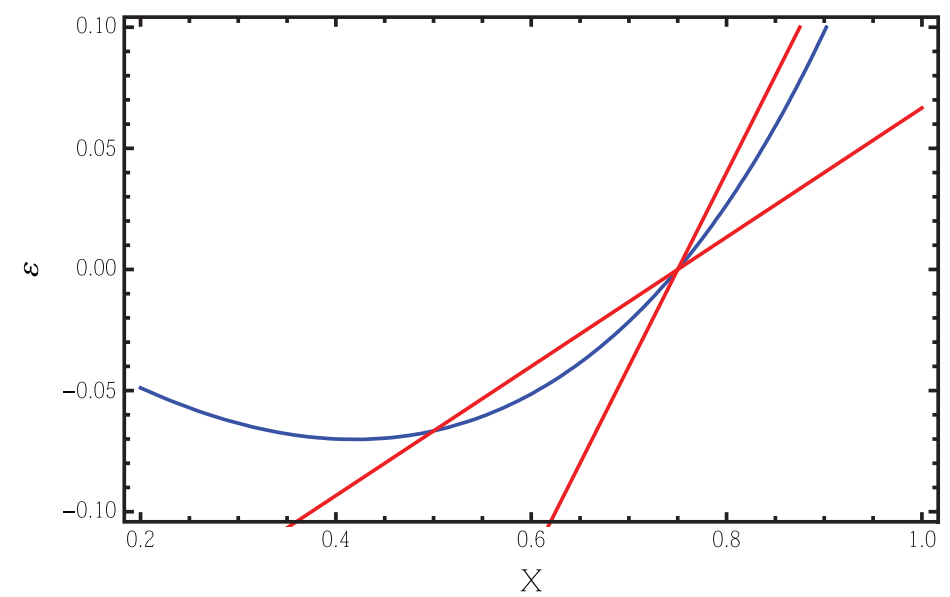

FIGURE 2. Existence of a pseudo-equilibrium of the sliding region. Red lines represent the boundaries of the sliding region as functions of $x$. The blue curve is the solution to equation (4.8).

Both the upper and lower bounds are positive for $\varepsilon_{0}<\varepsilon<0$, implying that the pseudoequilibrium is unstable. So, in this parameter range, the pseudo-equilibrium is totally unstable.

Theorem 4.2 For $\varepsilon<\varepsilon_{0}$, there exists one globally stable, real equilibrium and no pseudoequilibria.

Proof First, the location of the equilibrium for the region $k=1$ is given by (4.2), which shows that this equilibrium is real in the specified parameter range. The stability of the equilibrium is not dependent on $\varepsilon$, and has eigenvalues

$$
\lambda=-2
$$

and

$$
\lambda=-\frac{3}{2}
$$

indicating stability. The equilibrium for the region $k=0$ remains virtual. So, it only remains to prove that there exists no pseudo-equilibria in this range. This is done using the same method as in Theorem 4.1, and again analysis shows that, for $\varepsilon<\varepsilon_{0}$, the $\varepsilon$ given by equation (4.8) is outside the range of the sliding region. So, no pseudo-equilibrium exists in this parameter range.

The local dynamics at $\varepsilon=\varepsilon_{0}$, corresponding to the border collision, are unique to non-smooth systems (see Figure 1). In the region $y>0$, all trajectories converge to the equilibrium. The eigenvectors are both transverse to the splitting manifold, and do not depend on $\varepsilon$, or the location of the equilibrium. However, in the region $y<0$, trajectories are repelled from the equilibrium, and are instead attracted to the virtual equilibrium of the $k=0$ system. In the direction along the sliding region, the filippov flow takes solutions 
away from the equilibrium. So, the equilibrium has two unstable directions, one along the splitting manifold, given by the Filippov flow, and the other transverse to the manifold, with infinite repulsion. One might call this equilibrium a centre of sorts; however, the location of the stable and unstable directions topologically disallow this situation in a smooth system.

However, this local picture is unsurprising in a non-smooth system, and is in fact typical of a border collision. What may be surprising is the change in stability of the equilibrium as the system passes through the bifurcation, which is intimately tied to the angle between the splitting manifold and the vector field in the $k=0$ region. If the vector field in the $k=0$ region were vertical, the equilibrium would not persist as a pseudo-equilibrium, and the structure of the bifurcation would be uninteresting. It should be reiterated that any talk about genericity of this type of bifurcation in a non-smooth system can not assume that the flow on one side of the splitting manifold can be made perpendicular to the manifold. If this were true, the local bifurcation seen here would not be possible.

\section{Global bifurcation structure}

The previous section discussed local dynamics as $\varepsilon=\varepsilon_{0}$, but the system also displays an interesting global bifurcation, as shown in Figure 3. At $\varepsilon=0$, a fused focus bifurcation occurs, which is a local bifurcation in which the sliding region changes from stable to unstable. As a result of this stability change, a periodic orbit appears, which grows as $\varepsilon$ becomes more negative. So, at $\varepsilon=\varepsilon_{0}$, this periodic orbit interacts with the equilibrium in a global bifurcation, which is a homoclinic implosion of sorts. For $\varepsilon>\varepsilon_{0}$, the system contains a large, stable periodic orbit, and an unstable pseudo-equilibrium. At $\varepsilon=\varepsilon_{0}$, the equilibrium is located on the splitting manifold, and has also collided with the periodic orbit, resulting in a continuum of homoclinic orbits from that point. Then, at $\varepsilon<\varepsilon_{0}$, the orbit structure is immediately destroyed, resulting in a single, globally stable, real equilibrium. The following theorems prove this scenario.

Theorem 5.1 For $\varepsilon_{0}<\varepsilon<0$, there exists a periodic orbit that intersects the line $y=0$ with $x$ values in the interval

$$
I=\left(\frac{1}{2}, \frac{3}{4}+\frac{15}{4} \varepsilon\right) .
$$

Proof We consider the return map of trajectories from $I$ to $I$, and show that the interval maps to a subset of itself. Then, we can immediately conclude the existence of a periodic orbit. This is, in fact, very simple to show. First, we note that trajectories with initial values in this interval return to the line $y=0$ in a bounded interval, with $x>0$. Moreover, all trajectories originating from the splitting manifold with

$$
x>\frac{3}{4}+\frac{15}{4} \varepsilon
$$

will intersect the splitting manifold again in $I$. This is a result of uniqueness of solutions in smooth dynamical systems, and a lack of invariant sets in the region $y>0$. The argument 


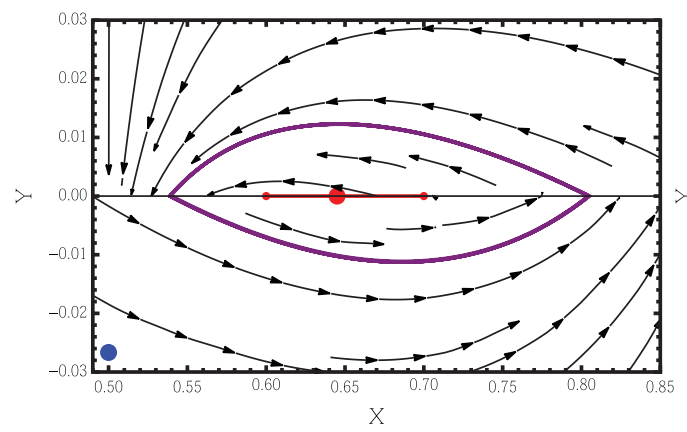

(a)

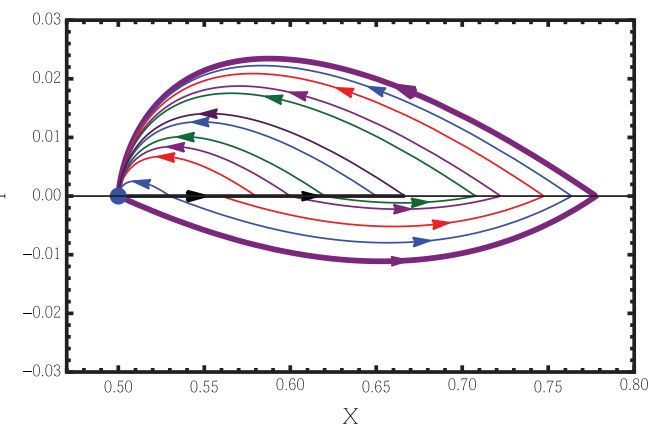

(b)

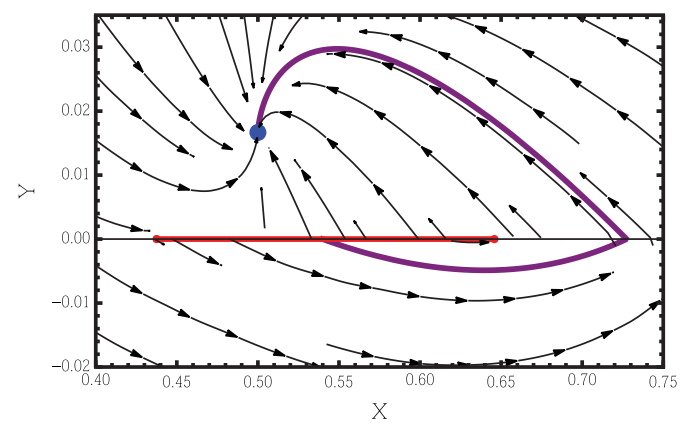

(c)

FIGURE 3. Global bifurcation structure of the boundary collision bifurcation. In (a), $\varepsilon=-\frac{1}{25}$, and the virtual equilibrium and unstable pseudo-node are shown as blue and red dots, respectively. The large stable periodic orbit exists. In (b), $\varepsilon=\varepsilon_{0}$, the bifurcation point. Infinitely, many homoclinic orbits occur. Each orbit slides arbitrarily along the sliding region, before leaving in either direction to eventually converge to the boundary equilibrium. The large periodic orbit becomes a homoclinic orbit from the boundary equilibrium. In (c), $\varepsilon=-\frac{1}{12}$, and the orbit structure has been destroyed. All trajectories are attracted to a globally stable, real equilibrium.

is three fold. For

$$
x>\frac{3}{4}+\frac{15}{4} \varepsilon
$$

and $\varepsilon_{0}<\varepsilon<0$,

$$
f(x, 0) \cdot\left(\begin{array}{l}
0 \\
1
\end{array}\right)>0
$$

meaning the vector field points away from the splitting manifold, so trajectories can't return to the splitting manifold to the right of the interval. The system in $y>0$ is also linear, and the equilibrium has a vertical eigenvector $\vec{e}_{2}$, so we immediately conclude the existence of a vertical trajectory along the line

$$
x=\frac{1}{2}
$$

converging to the virtual equilibrium. So, trajectories cannot return to the splitting manifold to the left of the interval. However, solutions are easily seen to be bounded, and 
the lack of invariant sets in the region $y>0$ indicates that solutions must return to the splitting manifold, indicating that the return map exists, and maps $I$ to itself. So, there exists a periodic orbit intersecting the splitting manifold in this interval.

One should note that this result immediately implies that the periodic orbit limits to an orbit through

$$
x=\frac{1}{2} \text {. }
$$

This is in fact a homoclinic orbit, co-incident to the equilibrium border collision.

Theorem 5.2 For $\varepsilon=\varepsilon_{0}$, there exists a homoclinic orbit through

$$
x=x_{0}=\frac{1}{2} \text {. }
$$

Proof There is a unique trajectory emanating from the equilibrium point $\left(x_{0}, 0\right)$ into the region $y<0$, based on the form of the $k=0$ system. This trajectory crosses the splitting manifold at some point $x>1$. All trajectories with initial condition $x>x_{0}, y>0$ converge to the equilibrium point without crossing the splitting manifold. Trajectories are not allowed to cross the vector field at any point $x>x_{0}$, because in that region, $\dot{y}>0$ along $y=0$. Moreover, trajectories can't cross the splitting manifold anywhere where $x<x_{0}$, because there exists a vertical line trajectory along $x=x_{0}$. Therefore, trajectories are squeezed from either side, and in particular the unique trajectory in the $k=0$ region, with initial condition $x=x_{0}$ must then return to $x=x_{0}$ after an infinite period, giving a homoclinic orbit.

Theorem 5.3 Using Filippov dynamics, when $\varepsilon=\varepsilon_{0}$, all orbits that slide along the splitting manifold are homoclinic orbits.

Proof First, because all trajectories in the $y>0$ region of phase space converge to the boundary equilibrium, it is clear that trajectories that travel arbitrarily along the sliding region and then escape to this region of phase space are homoclinic orbits. If an orbit leaves the splitting manifold into the $y<0$ region, it is constrained by the homoclinic orbit described in Theorem 5.2. Because there are no invariant sets in this region, these orbits will cross the splitting manifold, and then converge to the boundary equilibrium, and hence are also homoclinic orbits. See Figure 3 for an illustration of these orbits.

\section{The corresponding smooth picture}

There are several key differences between this non-smooth homoclinic bifurcation and a standard homoclinic bifurcation, which indicate that this phenomenon is not a limit of a smooth homoclinic bifurcation. The bifurcation does not leave behind a saddle equilibrium point. The homoclinic orbits are space filling. Most importantly, there is a shear in the stable and unstable directions of the equilibrium on the homoclinic orbit. 


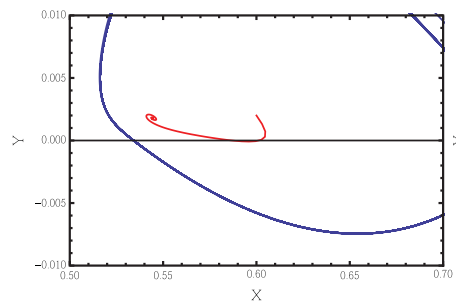

(a)

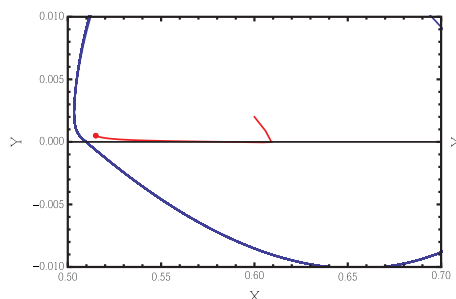

(d)

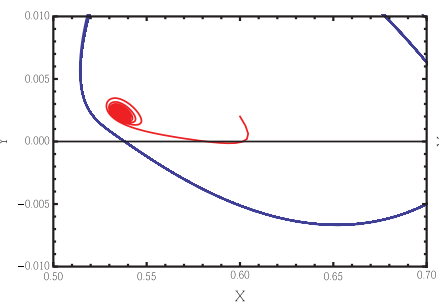

(b)

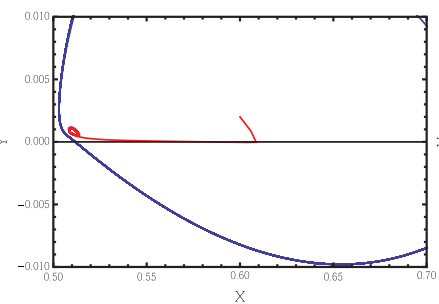

(e)

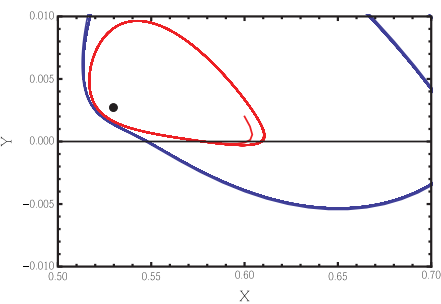

(c)

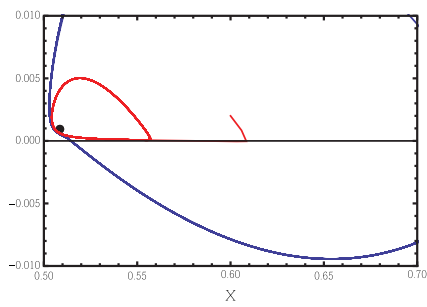

(f)

FIGURE 4. The smooth system as it passes through the bifurcation. A subcritical Hopf bifurcation and a periodic orbit saddle node bifurcation limit to a single bifurcation point as $a \rightarrow 0$. Top row: $a=10^{-3}, \mathrm{~A}: \varepsilon=-0.0633 \mathrm{~B}: \varepsilon=-0.0650 \mathrm{C}: \varepsilon=-0.0662$. Bottom row $: a=10^{-4}, \mathrm{D}: \varepsilon=-0.0658$ $\mathrm{E}: \varepsilon=-0.0665 \mathrm{~F}: \varepsilon=-0.0668$.

Alternatively, the system shows some similarities to canard orbits. The homoclinic orbits are of all sizes, as if there is a canard explosion occurring at a single point. Moreover, the aftermath of the non-smooth bifurcation is attraction to a larger periodic structure, analogous to a relaxation oscillation. However, the model does not have the bi-stability inherent to canard systems. So, one wonders which smooth phenomena might limit to this non-smooth homoclinic bifurcation.

In this system, we can answer this question directly, because Welander formulated two versions of his model, the non-smooth system being the pointwise limit of a smooth system (See Figure 4.). In the smooth system, instead of $k$ being a non-smooth Heaviside function,

$$
k=\frac{1}{\pi} \tan ^{-1}\left(\frac{y}{a}\right)+\frac{1}{2} .
$$

Therefore, we can describe which behaviour in the smooth model leads to such unusual behaviour in the non-smooth model. Proving that the smooth phenomena limit to the homoclinic bifurcation is non-trivial; however, simulations indicate that this is the case.

The smooth system contains a supercritical Hopf bifurcation, which is a direct analogue to a similar phenomenon in the non-smooth system. However, the smooth system also contains a subcritical Hopf bifurcation in the parameter vicinity of $\varepsilon=\varepsilon_{0}$. The stable and unstable periodic orbits from these bifurcations grow until they annihilate each other in a periodic orbit saddle node bifurcation, leaving behind a globally stable equilibrium point. The subcritical Hopf bifurcation and the periodic orbit saddle node limit to the same parameter value as $a \rightarrow 0$, and the system becomes non-smooth. 


\section{Discussion}

There are a few interesting implications of this result. First, it is a testament to the complexities possible in non-smooth systems, even in the simple case of equilibria colliding with a splitting manifold. Consideration of many systems is necessary before any generalizations can be made about classification of behaviour in these systems. In particular, previous classification systems have missed details of both the local and global behaviour for the border collision bifurcation. There are several interesting co-dimension one phenomena in Welander's model; however, a full bifurcation analysis of the model has not been undertaken. In addition, one can consider the non-smooth version of Welander's model as a limiting case of a smooth system, in which case the co-dimension one phenomena of the border collision are a limiting case of the co-dimension two phenomenon. For different values of the parameter $a$, the bifurcation branches into a subcritical Hopf bifurcation and a periodic orbit saddle node, as discussed in Section 6. It would be interesting to determine which other non-standard Filippov bifurcation phenomena are likewise limiting cases of more standard smooth bifurcations, although the framework for answering such a question is currently not fully developed. Additionally, it is possible that with inclusion of the other two model parameters, the system contains bifurcations like the Takens-Bogdanov bifurcation, which contains some of the features seen in this analysis. More analysis is necessary to determine if this is the case. Moreover, a more general relationship between smooth and non-smooth systems is not clear. In this system, the non-smooth structure is qualitatively different from the smooth structure. In fact, even slightly away from the bifurcation point, the periodic orbit, which is unique in the non-smooth system, does not perturb to a unique periodic orbit in the smooth system. Instead, the smooth system has two periodic orbits, one stable and one unstable. This is in spite of the nice (monotone) behaviour of the smooth approximation to the Heaviside function. It is possible that the homoclinic explosion is a fundamentally non-smooth phenomenon, which is the limiting behaviour of many different smooth behaviours. So, this is yet another indication that care must be taken when using non-smooth approximations to smooth systems.

\section{Acknowledgements}

The author would like to thank Richard McGehee, Mike Jeffrey, Paul Glendinning and Mary Silber for their input and advice on this work.

\section{References}

[1] Buzzi, C. A., Da Silva, P. R. \& Teixeira, M. A. (2006) A singular approach to discontinuous vector fields on the plane. J. Differ. Equ. 231(2), 633-655.

[2] Colombo, A., di Bernardo, M., Hogan, S. J. \& Jeffrey, M. R. (2012) Bifurcations of piecewise smooth flows: Perspectives, methodologies and open problems. Phys. D: Nonlinear Phenom. 241(22), 1845-1860.

[3] Dercole, F., Gragnani, A. \& Rinaldi, S. (2007) Bifurcation analysis of piecewise smooth ecological models. Theor. Popul. Biol. 72(2), 197-213. 
[4] Di Bernardo, M., Budd, C., Champneys, A. R. \& KowalczyK, P. (2008) Piecewise-Smooth Dynamical Systems: Theory and Applications, vol. 163, Springer Science and Business Media, London, UK.

[5] di Bernardo, M., Nordmark, A. \& Olivar, G. (2008) Discontinuity-induced bifurcations of equilibria in piecewise-smooth and impacting dynamical systems. Phys. D: Nonlinear Phenom. 237(1), 119-136.

[6] Eisenman, I. \& Wettlaufer, J. S. (2009) Nonlinear threshold behavior during the loss of arctic sea ice. Proc. Natl. Acad. Sci. USA 106(1), 28-32.

[7] Filippov, A. F. \& ArscotT, F. M. (1988) Differential Equations with Discontinuous Righthand Sides: Control Systems, vol. 18, Springer Science and Business Media, Dordrecht, Netherlands.

[8] Guardia, M., Seara, T. M. \& Teixeira, M. A. (2011) Generic bifurcations of low codimension of planar filippov systems. J. Differ. Equ. 250(4), 1967-2023.

[9] Hill, K., Аввот, D. S. \& Silber, M. (2015) Analysis of an arctic sea ice loss model in the limit of a discontinuous albedo. SIAM J. Appl. Dynamical Syst. 15(2), 1163-1192. arXiv:1509.00059.

[10] JefFrey, M. R. (2011) Nondeterminism in the limit of nonsmooth dynamics. Phys. Rev. Lett. 106(25), Article no. 254103.

[11] Jefretey, M. R. (2014) Hidden dynamics in models of discontinuity and switching. Phys. D: Nonlinear Phenom. 273, 34-45.

[12] Kristiansen, K. U. \& Hogan, S. J. (2015) Regularizations of two-fold bifurcations in planar piecewise smooth systems using blow up. SIAM J. Appl. Dyn. Syst. 14(4), 17311786. arXiv:1502.06210.

[13] Kuznetsov, Y. A., Rinaldi, S. \& Gragnani, A. (2003) One-parameter bifurcations in planar filippov systems. Int. J. Bifurc. Chaos 13(8), 2157-2188.

[14] Llibre, J., Da Silva, P. R. \& Teixeira, M. A. (2009) Study of singularities in nonsmooth dynamical systems via singular perturbation. SIAM J. Appl. Dyn. Syst. 8(1), 508-526.

[15] Makarenkov, O. \& Lamb, J. S. W. (2012) Dynamics and bifurcations of nonsmooth systems: A survey. Phys. D: Nonlinear Phenom. 241(22), 1826-1844.

[16] Sotomayor, J. \& Teixeira, M. A. (1996) Regularization of discontinuous vector fields. In: International Conference on Differential Equations, Lisboa, Portugal, pp. 207-223.

[17] Stommel, H. (1961) Thermohaline convection with two stable regimes of flow. Tellus 13(2), 224-230.

[18] Teixeira, M. A. \& DA Silva, P. R. (2012) Regularization and singular perturbation techniques for non-smooth systems. Phys. D: Nonlinear Phenom. 241(22), 1948-1955.

[19] Welander, P. (1982) A simple heat-salt oscillator. Dyn. Atmos. 6(4), 233-242.

[20] Welander, P. (1986) Thermohaline effects in the ocean circulation and related simple models. NATO ASI Ser. C: Math. Phys. Sci. 190, pp. 163-200. 